„This is the peer reviewed version of the following article: Chris Guhrenz, Vladimir Sayevich, Florian Weigert, Eileen Hollinger, Annett Reichhelm, Ute Resch-Genger, Nikolai Gaponik and Alexander Eychmüller (2017). Transfer of Inorganic-Capped Nanocrystals into Aqueous Media. Journal of Physical Chemistry Letters, 2017, 8 (22), pp 5573-5578, which has been published in final form at DOI:10.1021/acs.jpclett.7b02319. “

\title{
Transfer of Inorganic-Capped Nanocrystals into
}

\section{Aqueous Media}

Chris Guhrenz,,$^{\dagger}$ Vladimir Sayevich, ${ }^{\dagger}$ Florian Weigert, ${ }^{*}$ Eileen Hollinger, ${ }^{\dagger}$ Annett Reichhelm, ${ }^{\dagger}$ Ute Resch-Genger, Nikolai Gaponik, ${ }^{\dagger, *}$ and Alexander Eychmüller ${ }^{\dagger}$

${ }^{\dagger}$ Physical Chemistry, Technische Universität Dresden, Bergstr. 66b, 01062 Dresden, Germany ${ }^{\ddagger}$ BAM Federal Institute for Materials Research and Testing, Division Biophotonics, RichardWillstätter Str. 11, 12489 Berlin, Germany

\section{Corresponding Author}

*E-mail: Nikolai.Gaponik@chemie.tu-dresden.de 


\section{ABSTRACT}

Herein, we report on a novel and simple approach to surface ligand design of CdSe-based nanocrystals (NCs) with biocompatible, heterobifunctional polyethylene glycol (PEG) molecules. This method provides high transfer yields of the NCs into aqueous media with preservation of the narrow and symmetric emission bands of the initial organic-capped NCs regardless of their interior crystal structure and surface chemistry. The PEG-functionalized NCs show small sizes, high photoluminescence quantum yields of up to $75 \%$ as well as impressive optical and colloidal stability. This universal approach is applied to different fluorescent nanomaterials $\left(\mathrm{CdSe} / \mathrm{CdS}, \mathrm{CdSe} / \mathrm{CdSCd}_{\mathrm{x}} \mathrm{Zn}_{1-\mathrm{x}} \mathrm{S}\right.$, and $\left.\mathrm{CdSe} / \mathrm{CdS} / \mathrm{ZnS}\right)$ extending the great potential of organic-capped NCs for biological applications.

\section{TOC GRAPHIC}

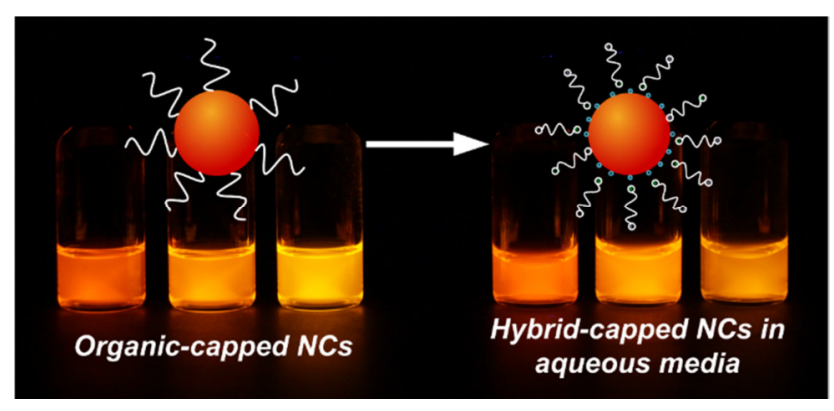

KEYWORDS: Ligand exchange, ligand design, hybrid capping, phase transfer, polyethylene glycol, bioconjugation. 
Surface capping ligands - molecules or ionic species that bind coordinatively to the surface of nanocrystals (NCs) - largely define the chemical characteristics of colloidal nanomaterials. ${ }^{1-3}$ The well-developed routes for NC synthesis in organic solvents mainly use hydrophobic ligands with long hydrocarbon chains. These surfactants control the nucleation and growth dynamics of the NCs, can saturate surface dangling bonds, as well as provide the chemical and colloidal stability and solubility of the NCs in organic solvents and polymers.

Many applications of NCs and related processing steps require, however, dispersibility in other matrices and hence, post-synthetic strategies of ligand design of organic-capped NCs. Particularly, the replacement of the initial organic molecules with inorganic species via ligand exchange enables to prepare $\mathrm{NC}$-based solids with tunable electronic properties required for solid-state devices such as field-effect transistors (FETs), photodetectors, thermoelectrics, and solar cells. ${ }^{4-13}$ Surface functionalization with specific hydrophilic molecules is necessary to utilize the unique optical and physicochemical properties of colloidal NCs for biomedical applications (e.g. for cell targeting, cell imaging, sensing, and drug delivery). ${ }^{14-20}$

To prepare biocompatible NCs, the pristine hydrophobic ligands can either be encapsulated e.g. with an amphiphilic polymer or replaced with hydrophilic molecules like mercaptoundecanoic acid, dihydrolipoic acid (DHLA), or cysteine. ${ }^{14,15,20-24}$ In the first approach, block copolymers or phospholipid coatings are used. This minimizes post-synthetic changes in the NC properties as the NCs preserve their non-functional hydrocarbon shell and the ligand-tosurface atom bonds are not disrupted, yet the effective NC size is considerably increased to a few ten $\mathrm{nm}$. This can be critical for all studies where NC size matters like cellular uptake and bioimaging experiments. The second approach addresses the need for a compact capping layer and a small size for the resulting particle architecture by exchanging the initial hydrophobic 
surface ligands with hydrophilic molecules. Ligand replacement is, however, usually accompanied by a strong decrease in the photoluminescence quantum yield (PL QY), deteriorating optical performance and limit of detection, and sometimes even by structural modifications of the ligand shell layers. ${ }^{25-27} \mathrm{~A}$ widely used approach to NC phase transfer utilizes small molecules with one thiol functional group like cysteine. These molecules can suffer from photoinduced dimerization yielding disulfides, thereby limiting NC colloidal stability. To overcome these challenges, the surface modification of the NCs with stronger binding dithiolterminated DHLA ligands provides enhanced stability and the additional connection to polyethylene glycol (PEG) molecules guarantees solubility in a wide $\mathrm{pH}$ range. ${ }^{28,29}$ Furthermore, these DHLA-PEG ligands form self-assembled monolayers on the NC surface and their functional end groups can be easily used for bioconjugation.

The functionalization with PEG molecules is usually performed in two steps: i) ligand exchange of the pristine molecules of the NCs with a short chain polar stabilizer like cysteine and ii) stabilizer replacement with a stronger binding, thiol-containing PEG derivate. ${ }^{30,31}$ This ligand exchange procedure strongly depends on the NC surface chemistry and is commonly done with NCs with a zinc-terminated surface and tri- $n$-octylphosphine/tri- $n$-octylphosphine oxide (TOP/TOPO) ligands and cannot be easily adapted to NCs with other capping species and metalenriched surfaces. More importantly, it entails low transfer yields of the NCs and is often accompanied by a considerable drop in PL QY. Investigations towards the solubilization of $\mathrm{InP} / \mathrm{ZnS}$ NCs via a two-phase system with cysteine as new ligand showed e.g. a low transfer yield of $60 \%$ and a strong decrease of the PL QY to $4 \% .{ }^{27}$

Here, we present a novel approach to ligand design for preparing biocompatible NCs utilizing the intermediate capping of NCs with tiny inorganic ligands, currently used for the preparation of 
advanced electronic and optoelectronic devices. ${ }^{4-6,8-13}$ This surface modification is already applicable with high transfer yields for a broad range of NCs with different surface chemistry. ${ }^{4,5,8,32}$ The resulting all-inorganic NCs are colloidally stable in polar solvents but not in aqueous media. Recently, we found that amine molecules can replace the positively charged labile diffusion region around the particles and transfer these all-inorganic NCs into non-polar solvents. ${ }^{11}$ The extension of this approach could be a reliable basis for a universal ligand design procedure used for the solubilization of organic-capped NCs in aqueous media. Using heterobifunctional PEG (HS-PEG-NH 2 ) ligands instead of short-chained amine molecules allows a biocompatible functionalization of the NCs.

This novel technique is based on a three-step procedure (Figure 1). First, the initial organic ligands of the NCs were exchanged with chloride ions, leading to all-inorganic-capped NCs which are stable in high polar solvents ( $N$-methylformamide, MFA). Chlorine-based species are known not to introduce deep trap levels and, in some cases, can provide surface passivation as shown for CdTe NCs. ${ }^{33}$ Second, the subsequent functionalization of these chlorido-capped NCs with short-chain $N$-butylamine molecules enable a fast and quantitative transfer of the NCs from polar (MFA) into non-polar media (chloroform). Finally, the labile $N$-butylamine ligand layer is replaced with bifunctional HS-PEG-NH$H_{2}$ molecules showing a stronger binding affinity to the NC surface than the short amines and introducing a good water dispersibility. This strategy provides high transfer yields and preserves the narrow and symmetric emission bands and high PL QYs of the NCs. The biocompatible NCs show an impressive colloidal stability with no sign of aggregation revealed by dynamic light scattering (DLS) measurements and transmission electron microscopy (TEM). 


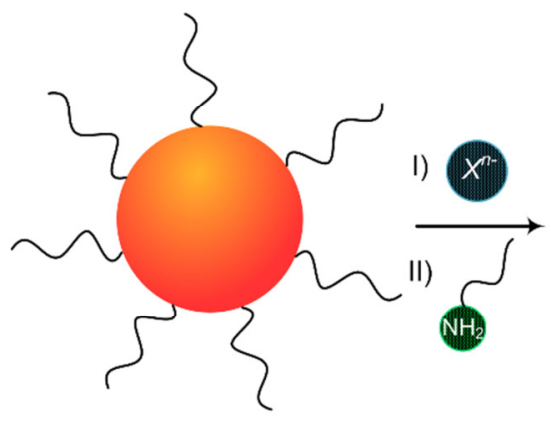

Organic-capped NCs

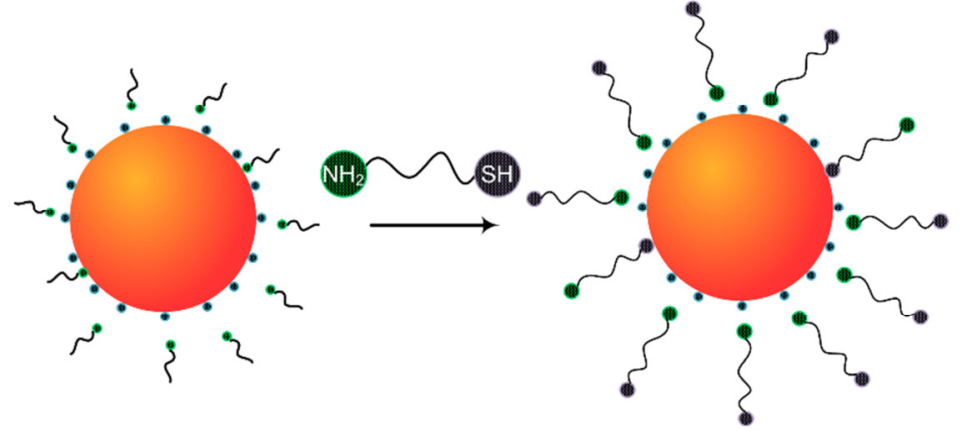

Hybrid-capped NCs in organic media
Hybrid-capped NCs in aqueous media

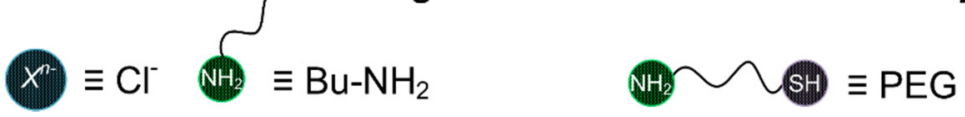

Figure 1. Scheme of the surface modification of the initially organic-capped NCs. The ligand exchange procedure includes i) an all-inorganic capping of the NCs with chloride ions and ii) phase transfer to non-polar media using $N$-butylamine molecules. Subsequent addition of heterobifunctional HS-PEG-NH $\mathrm{N}_{2}$ molecules replaces the short-chain amines and provides water dispersibility.

In order to emphasize the versatility of this procedure, three representative CdSe-based NCs with typical, yet sufficiently different chemical composition of the inorganic passivation shell and, hence, also material-dependent shape of the confinement potential were used: i) CdSterminated core/shell $\mathrm{CdSe} / \mathrm{CdS}$ NCs with 8 monolayers (ML) of $\mathrm{CdS}$, ii) alloyed $\mathrm{CdSe} / \mathrm{CdSCd}_{\mathrm{x}} \mathrm{Zn}_{1-\mathrm{x}} \mathrm{S}$ NCs with $7 \mathrm{ML} \mathrm{CdS}$ and a thin external $\mathrm{ZnS}$ shell (1 ML), and iii) core/shell/shell CdSe/CdS/ZnS (7/3 ML) NCs with a ZnS-terminated surface. Using the same CdSe NC cores with a size of $2.7 \mathrm{~nm}$, the particles were shelled with slight variations according to protocols published by Chen et al. ${ }^{34}$ and Boldt et al. ${ }^{35}$ applying a continuous shell precursor infusion method (for further details, see Supporting Information (SI)). With the aid of a temperature ramp during the shelling process, we could prevent the commonly observed 
reduction of the PL QY after ZnS shelling. The resulting CdSe/CdS core/shell NCs showed a PL QY of $65 \%$ while the PL QYs of the ZnS-terminated particles reached $82 \%$ and $93 \%$ for alloyed $\mathrm{CdSe} / \mathrm{CdSCd}_{\mathrm{x}} \mathrm{Zn}_{1-\mathrm{x}} \mathrm{S}$ and $\mathrm{CdSe} / \mathrm{CdS} / \mathrm{ZnS}$ NCs, respectively. The corresponding PL spectra (see SI, Figure S1) revealed symmetric and narrow emission bands and a small PL broadening after growing a thick $\mathrm{ZnS}$ shell (FWHM of $136 \mathrm{meV}$ ) in comparison to the pure $\mathrm{CdSe} / \mathrm{CdS} \mathrm{NCs}$ (115 $\mathrm{meV}$ ) as previously observed by Boldt et al. ${ }^{35}$ TEM images of the NCs are shown in the SI (Figure S2), underlining the narrow size distribution of all samples.

Subsequently, the surface of the NCs was modified i) with chloride ions and ii) $N$-butylamine which was added for hybrid capping and phase transfer from MFA to chloroform as described previously. ${ }^{11}$ In this paper, we successfully functionalized Cd- and Zn-terminated NCs with chloride ions by means of properly adjusted reaction conditions (for further details, see SI). In the case of the $\mathrm{ZnS}$-shelled NCs, we observed a small red shift in the absorption and emission spectra $\left(2 \mathrm{~nm}\right.$ for the emission band of $\mathrm{CdSe} / \mathrm{CdSCd}_{\mathrm{x}} \mathrm{Zn}_{1-\mathrm{x}} \mathrm{S}$ NCs and $8 \mathrm{~nm}$ for the $\mathrm{CdSe} / \mathrm{CdS} / \mathrm{ZnS} \mathrm{NCs}$, respectively). This could be attributed to a change in the surrounding medium and/or to interdiffusion of chloride ions into the $\mathrm{Zn}$-containing lattice of the NC shell during the phase transfer between the immiscible phases of MFA and $n$-hexane within 12 hours. Generally, the PL QYs of the NCs are reduced after this two-step surface modification procedure to $31-54 \%$ of the initial values in $n$-hexane (Table 1), but the size of the NCs (Figure 2, left; see SI, Figure S3) and the PL spectra (Figure 4) remain unaffected. In addition, DLS measurements (Figure 2, right) reveal that the hydrodynamic diameters of the chloride/ $N$-butylamine-capped NCs in chloroform closely match the initially determined size of the NCs using TEM (see SI; Figure S2) and just negligible amounts of NC agglomerates are observed. This underlines that 
surface functionalization with short amine ligands allowed for high transfer yields $(>90 \%)$ with preservation of high PL QYs and colloidal stability.
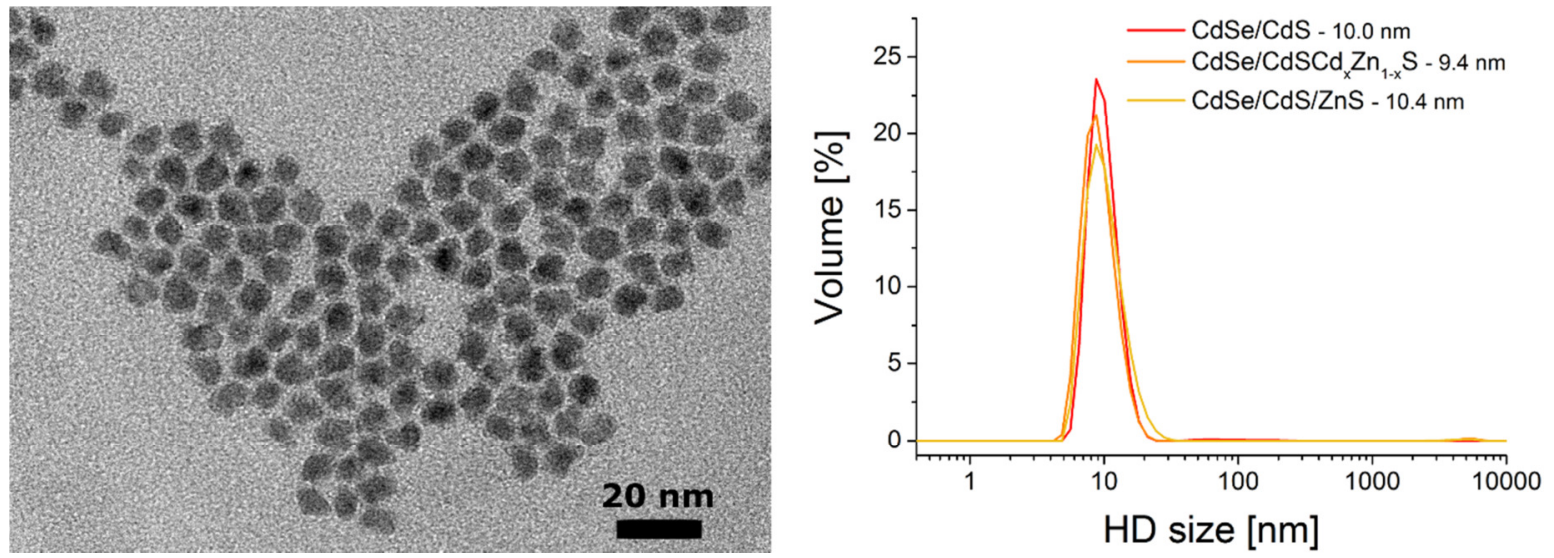

Figure 2. Representative TEM image (left) of ZnS-terminated core/shell/shell chloride/N butylamine hybrid-capped CdSe/CdS/ZnS (6/3 ML) NCs and DLS data (right) of the surface modified hybrid-capped NCs in chloroform.

The hybrid-capped core/shell(/shell) NCs are finally transferred into aqueous solution with the aid of heterobifunctional PEG molecules. For this purpose, the HS-PEG-NH $\mathrm{N}_{2}$ ligands are dissolved in chloroform, the short-chain chloride/ $N$-butylamine-capped NCs are added, the chloroform is evaporated under reduced pressure, and the dried particles are redispersed in water. By using this procedure, all samples are successfully transferred into water. In the representative TEM image in Figure 3, well-separated chloride/PEG-NH${ }_{2}$ hybrid-capped CdSe/CdS NCs can be observed. The hydrodynamic diameter of $\mathrm{CdSe} / \mathrm{CdS}$ and alloyed $\mathrm{CdSe} / \mathrm{CdSCd}_{\mathrm{x}} \mathrm{Zn}_{1-\mathrm{x}} \mathrm{S} \mathrm{NCs}$ amounts to $\sim 18.0 \mathrm{~nm}$ and $\sim 21.0 \mathrm{~nm}$, respectively, as obtained from DLS measurements (Figure 3, right). This is comparable with data published by Chen et al. for a direct ligand exchange of CdSe/CdS NCs with methoxy-PEG thiol. ${ }^{34}$ Nevertheless, that method suffers from a low transfer yield according to our experience and, due to the lack of a reactive end group, it is difficult to 
conjugate these PEGylated NCs with peptides, proteins or nucleic acids. Following our strategy also alloyed- and ZnS-terminated NCs can be phase transferred. For the latter one higher hydrodynamic diameters with minor amounts of small agglomerates are observed. Nevertheless, TEM images reveal a separation of the core/shell/shell NCs (see SI, Figure S4).

The applied HS-PEG-NH $\mathrm{N}_{2}$ capping ligands present ambidentate molecules with $\mathrm{N}$ - and Sbound coordination functional modes. Our recent studies showed that amine groups can bind to the negatively charged chlorido-terminated NC surface under formation of dense hydrophobic monolayers. ${ }^{11}$ On the other hand, it is known that thiol groups show high binding affinity to Cdand Zn-enriched NC surfaces, implying additional coordination via S-bound modes. Keeping in mind that PEG-functionalized NCs form stable colloidal solutions in basic media ( $\mathrm{pH}>7$, see $\mathrm{SI}$ ), it is reasonable to expect the preferable surface coordination with amine groups, while the outer deprotonated thiolate provides electrostatic repulsion and stabilization of the NCs in polar aqueous media. In conjunction with hydrophobic interactions between the PEG chains and sterical stabilization, this leads to a fast surface modification of the hybrid-capped chloride/ $\mathrm{N}$ butylamine NCs as well as to a better NC surface passivation. The latter is derived from the enhanced PL QYs of the PEG-stabilized NCs, confirmed by both steady-state and time-resolved spectroscopy (Table 1 and SI, Figure S5).
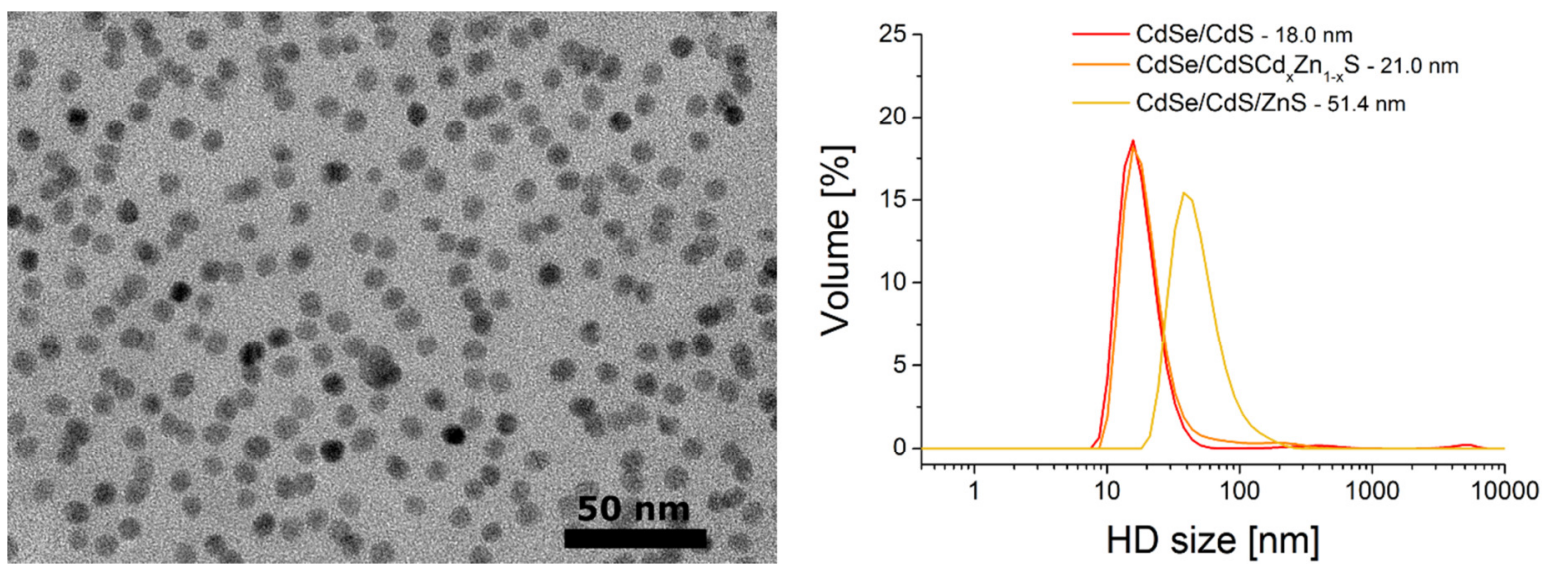
Figure 3. TEM image of phase-transferred chloride/PEG- $\mathrm{NH}_{2}$ hybrid-capped $\mathrm{CdSe} / \mathrm{CdS} \mathrm{NCs}$ in aqueous solution (left) and the corresponding DLS data of the phase-transferred NCs (right).

While the narrow PL emission band of the NCs is not affected by the surface modification with HS-PEG-NH $\mathrm{N}_{2}$ (Figure 4), the diminution in the PL QY is only relatively moderate (Table 1) with values of $39 \%$ and even $74 \%$ and $75 \%$ resulting for thick-shell $\mathrm{CdSe} / \mathrm{CdS} \mathrm{NCs}$ and core/shell/shell NCs with alloyed- and pure ZnS-termination. This equals $60 \%, 90 \%$ and $81 \%$ of the initial values in $n$-hexane, respectively. The PL QY enhancement compared to capping with short and labile $N$-butylamine ligands is attributed to a better passivation of the NC surface by HS-PEG-NH $\mathrm{N}_{2}$. Variations in the degree of PL QY loss after phase transfer most likely reflect differences in the surface passivation of the CdSe cores. It is well-known that a $\mathrm{ZnS}$ layer around $\mathrm{CdSe} / \mathrm{CdS}$ NCs provides a deeper potential leading to a more efficient confinement of electrons and holes in the core and spatial separation from dangling bonds or excess charges at the NC surface. Additionally, the intermediate CdS layer reduces the larger lattice mismatch between CdSe and $\mathrm{ZnS}$ reducing interfacial strain.
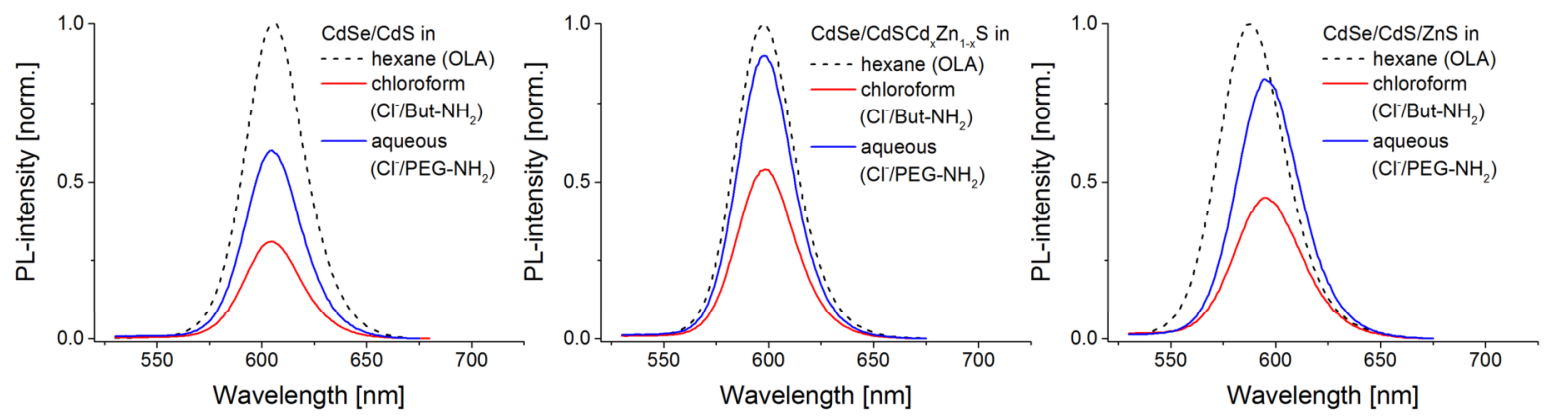

Figure 4. Normalized PL spectra of the initial NCs in $n$-hexane (dotted lines) and the PL spectra of the hybrid-capped NCs in chloroform (red lines; surface ligands chloride/ $N$ butylamine) and in aqueous solution (blue lines; surface ligands chloride/PEG- $\mathrm{NH}_{2}$ ). 
Table 1. PL QY values of the initial NCs in $n$-hexane as well as the chloride/ $N$-butylamineand chloride/PEG-NH 2 -functionalized hybrid-capped NCs in chloroform and water, respectively.

\begin{tabular}{|c|c|c|c|c|}
\hline Sample & Solution & $\begin{array}{l}\text { Capping } \\
\text { ligand }\end{array}$ & $\begin{array}{c}\text { PL QY } \\
{[\%]}\end{array}$ & $\begin{array}{c}\text { PL QY preservation } \\
{[\%]}\end{array}$ \\
\hline \multirow{3}{*}{$\mathrm{CdSe} / \mathrm{CdS}$} & hexane & OLA & 65 & \\
\hline & chloroform & $\mathrm{Cl}^{-} / \mathrm{But}_{-} \mathrm{NH}_{2}$ & 20 & 31 \\
\hline & water & $\mathrm{Cl}^{-} / \mathrm{PEG}-\mathrm{NH}_{2}$ & 39 & 60 \\
\hline \multirow{3}{*}{$\mathrm{CdSe} / \mathrm{CdSCd} \mathrm{Z}_{\mathrm{x}} \mathrm{Zn}_{1-\mathrm{x}} \mathrm{S}$} & hexane & OLA & 82 & \\
\hline & chloroform & $\mathrm{Cl}^{-} /$But- $\mathrm{NH}_{2}$ & 44 & 54 \\
\hline & water & $\mathrm{Cl}^{-} / \mathrm{PEG}-\mathrm{NH}_{2}$ & 74 & 90 \\
\hline \multirow{3}{*}{$\mathrm{CdSe} / \mathrm{CdS} / \mathrm{ZnS}$} & hexane & OLA & 93 & \\
\hline & chloroform & $\mathrm{Cl}^{-} /$But- $\mathrm{NH}_{2}$ & 42 & 45 \\
\hline & water & $\mathrm{Cl}^{-} / \mathrm{PEG}-\mathrm{NH}_{2}$ & 75 & 81 \\
\hline
\end{tabular}

These trends in PL QY values comply well with the changes in the PL decay kinetics of the NCs before and after ligand exchange (see SI, Figure S5). In general, the PL decay of the organic- and PEG-capped NCs are not mono-exponential. The surface modification of the CdSand ZnS-terminated core/shell(/shell) NCs leads to a greater contribution of shorter lived components especially for CdSe/CdS NCs, reflecting the observed trend in changes of PL QY. According to $\mathrm{Pu}$ et al. this behavior can be related to deep trap states that are not fully passivated by chloride or PEG molecules. ${ }^{36}$ An opposed behavior is observed for the alloyed $\mathrm{CdSe} / \mathrm{CdSCd} \mathrm{Zn}_{1-\mathrm{x}} \mathrm{S}$ NCs. While the lifetime of the short lived decay components before and after ligand exchange is barely affected, the lifetime of the longer lived decay component is increased by PEG capping, which can be related to a higher concentration of shallow surface 
traps. In all cases, PEG molecules cannot completely passivate the dangling bonds and surface trap states in both core/shell(/shell) and alloyed NCs.

In summary, we demonstrate a versatile phase transfer method for organic-capped NCs from non-polar to aqueous solution via hybrid-capped chloride/ $N$-butylamine NCs and applying heterobifunctional HS-PEG- $\mathrm{NH}_{2}$ molecules. This fast three-step ligand exchange procedure enables high transfer yields, prevents largely NC agglomeration, and preserves the initially high PL QYs of high quality NCs synthesized by hot injection methods. In addition, it is applicable to various $\mathrm{NC}$ surfaces (pure $\mathrm{CdS}, \mathrm{ZnS}$, and alloyed $\mathrm{Cd}_{\mathrm{x}} \mathrm{Zn}_{1-\mathrm{x}} \mathrm{S}$ ) under preservation of the colloidal stability ( $>2$ months) of the hybrid-capped NC solutions in chloroform and water and of the NC size, as can be seen from the provided DLS data and TEM images. After PEGylation the NCs show a strong recovery of PL QY (up to 75\%) and PL lifetimes which are comparable with the initial values of the organic-capped NCs. This ligand exchange strategy opens a simple and versatile pathway for various well-known $\mathrm{NC}$ surfaces and make them usable for various applications, e.g. in electronic devices (hybrid capping with $N$-butylamine), bioconjugation (hybrid capping with $\mathrm{HS}-\mathrm{PEG}-\mathrm{NH}_{2}$ ) and bioimaging.

\section{ASSOCIATED CONTENT}

Supporting Information. Synthesis and ligand exchange procedures, absorption and PL spectra, time-resolved PL decay, TEM images of the initial and hybrid-capped NCs.

\section{AUTHOR INFORMATION}

\section{Corresponding Author}

*E-mail: Nikolai.Gaponik@chemie.tu-dresden.de 


\section{Notes}

The authors declare no competing financial interests.

\section{ACKNOWLEDGMENT}

The authors gratefully acknowledge the financial support from the M-ERA.NET within the project of 'Integrated Computational Engineering, Characterization and Validation of Semiconductor Colloidal Nanocrystals with Advanced Properties' (ICENAP, GA 1289/3-1) and the ERC AdG AEROCAT. VS thanks Sächsische Aufbaubank, project 100315856 BacMot for financial support. In addition, the authors thank Susanne Goldberg for TEM measurements.

\section{REFERENCES}

(1) Talapin, D. V.; Lee, J.-S.; Kovalenko, M. V.; Shevchenko, E. V. Prospects of Colloidal Nanocrystals for Electronic and Optoelectronic Applications. Chem. Rev. 2010, 110, 389-458.

(2) Kovalenko, M. V.; Manna, L.; Cabot, A.; Hens, Z.; Talapin, D. V.; Kagan, C. R.; Klimov, V. I.; Rogach, A. L.; Reiss, P.; Milliron, D. J.; et al. Prospects of Nanoscience with Nanocrystals. ACS Nano 2015, 9, 1012-1057.

(3) Boles, M. A.; Ling, D.; Hyeon, T.; Talapin, D. V. The Surface Science of Nanocrystals. Nat. Mater. 2016, 15, 141-153.

(4) Kovalenko, M. V.; Scheele, M.; Talapin, D. V. Colloidal Nanocrystals with Molecular Metal Chalcogenide Surface Ligands. Science 2009, 324, 1417-1420.

(5) Nag, A.; Kovalenko, M. V.; Lee, J.-S.; Liu, W.; Spokoyny, B.; Talapin, D. V. Metal-Free Inorganic Ligands for Colloidal Nanocrystals: $\mathrm{S}^{2-}, \mathrm{HS}^{-}, \mathrm{Se}^{2-}, \mathrm{HSe}^{-}, \mathrm{Te}^{2-}, \mathrm{HTe}^{-}, \mathrm{TeS}_{3}{ }^{2-}, \mathrm{OH}^{-}$, and $\mathrm{NH}_{2}{ }^{-}$as Surface Ligands. J. Am. Chem. Soc. 2011, 133, 10612-10620. 
(6) Dong, A.; Ye, X.; Chen, J.; Kang, Y.; Gordon, T.; Kikkawa, J. M.; Murray, C. B. A Generalized Ligand-Exchange Strategy Enabling Sequential Surface Functionalization of Colloidal Nanocrystals. J. Am. Chem. Soc 2011, 133, 998-1006.

(7) Zhang, H.; Jang, J.; Liu, W.; Talapin, D. V. Colloidal Nanocrystals with Inorganic Halide, Pseudohalide, and Halometallate Ligands. ACS Nano 2014, 8, 7359-7369.

(8) Dolzhnikov, D. S.; Zhang, H.; Jang, J.; Son, J. S.; Panthani, M. G.; Shibata, T.; Chattopadhyay, S.; Talapin, D. V. Composition-Matched Molecular "Solders" for Semiconductors. Science 2015, 347, 425-428.

(9) Sayevich, V.; Gaponik, N.; Plötner, M.; Kruszynska, M.; Gemming, T.; Dzhagan, V. M.; Akhavan, S.; Zahn, D. R. T.; Demir, H. V.; Eychmüller, A. Stable Dispersion of Iodide-Capped PbSe Quantum Dots for High-Performance Low-Temperature Processed Electronics and Optoelectronics. Chem. Mater. 2015, 27, 4328-4337.

(10) Sayevich, V.; Guhrenz, C.; Sin, M.; Dzhagan, V. M.; Weiz, A.; Kasemann, D.; Brunner, E.; Ruck, M.; Zahn, D. R. T.; Leo, K.; et al. Chloride and Indium-Chloride-Complex Inorganic Ligands for Efficient Stabilization of Nanocrystals in Solution and Doping of Nanocrystal Solids. Adv. Funct. Mater. 2016, 26, 2163-2175.

(11) Sayevich, V.; Guhrenz, C.; Dzhagan, V. M.; Sin, M.; Werheid, M.; Cai, B.; Borchardt, L.; Widmer, J.; Zahn, D. R. T.; Brunner, E.; et al. Hybrid N-Butylamine-Based Ligands for Switching the Colloidal Solubility and Regimentation of Inorganic-Capped Nanocrystals. ACS Nano 2017, 11, 1559-1571.

(12) Fafarman, A. T.; Koh, W.-K.; Diroll, B. T.; Kim, D. K.; Ko, D.-K.; Oh, S. J.; Ye, X.; Doan-Nguyen, V.; Crump, M. R.; Reifsnyder, D. C.; et al. Thiocyanate-Capped Nanocrystal 
Colloids: Vibrational Reporter of Surface Chemistry and Solution-Based Route to Enhanced Coupling in Nanocrystal Solids. J. Am. Chem. Soc. 2011, 133, 15753-15761.

(13) Choi, J.-H.; Fafarman, A. T.; Oh, S. J.; Ko, D.-K.; Kim, D. K.; Diroll, B. T.; Muramoto, S.; Gillen, J. G.; Murray, C. B.; Kagan, C. R. Bandlike Transport in Strongly Coupled and Doped Quantum Dot Solids: A Route to High-Performance Thin-Film Electronics. Nano Lett. 2012, 12, $2631-2638$.

(14) Medintz, I. L.; Uyeda, H. T.; Goldman, E. R.; Mattoussi, H. Quantum Dot Bioconjugates for Imaging, Labelling and Sensing. Nat. Mater. 2005, 4, 435-446.

(15) Bilan, R.; Fleury, F.; Nabiev, I.; Sukhanova, A. Quantum Dot Surface Chemistry and Functionalization for Cell Targeting and Imaging. Bioconjug. Chem. 2015, 26, 609-624.

(16) Dubavik, A.; Sezgin, E.; Lesnyak, V.; Gaponik, N.; Schwille, P.; Eychmüller, A. Penetration of Amphiphilic Quantum Dots through Model and Cellular Plasma Membranes. ACS Nano 2012, 6, 2150-2156.

(17) Pelaz, B.; Alexiou, C.; Alvarez-Puebla, R. A.; Alves, F.; Andrews, A. M.; Ashraf, S.;

Balogh, L. P.; Ballerini, L.; Bestetti, A.; Brendel, C.; et al. Diverse Applications of Nanomedicine. ACS Nano 2017, 11, 2313-2381.

(18) Dubavik, A.; Lesnyak, V.; Thiessen, W.; Gaponik, N.; Wolff, T.; Eychmüller, A. Synthesis of Amphiphilic CdTe Nanocrystals. J. Phys. Chem. C 2009, 113, 4748-4750.

(19) Pellegrino, T.; Kudera, S.; Liedl, T.; Muñoz Javier, A.; Manna, L.; Parak, W. J. On the Development of Colloidal Nanoparticles towards Multifunctional Structures and Their Possible Use for Biological Applications. Small 2004, 1, 48-63.

(20) Bilan, R.; Nabiev, I.; Sukhanova, A. Quantum Dot-Based Nanotools for Bioimaging, Diagnostics, and Drug Delivery. ChemBioChem 2016, 17, 2103-2114. 
(21) Tyrakowski, C. M.; Snee, P. T. A Primer on the Synthesis, Water-Solubilization, and Functionalization of Quantum Dots, Their Use as Biological Sensing Agents, and Present Status. Phys. Chem. Chem. Phys. 2014, 16, 837-855.

(22) Mattoussi, H.; Palui, G.; Na, H. Bin. Luminescent Quantum Dots as Platforms for Probing in Vitro and in Vivo Biological Processes. Adv. Drug Deliv. Rev. 2012, 64, 138-166.

(23) Pelaz, B.; Jaber, S.; de Aberasturi, D. J.; Wulf, V.; Aida, T.; de la Fuente, J. M.; Feldmann, J.; Gaub, H. E.; Josephson, L.; Kagan, C. R.; et al. The State of Nanoparticle-Based Nanoscience and Biotechnology: Progress, Promises, and Challenges. ACS Nano 2012, 6, 84688483.

(24) Pellegrino, T.; Manna, L.; Kudera, S.; Liedl, T.; Koktysh, D.; Rogach, A. L.; Keller, S.; Rädler, J.; Natile, G.; Parak, W. J. Hydrophobic Nanocrystals Coated with an Amphiphilic Polymer Shell: A General Route to Water Soluble Nanocrystals. Nano Lett. 2004, 4, 703-707.

(25) Liu, W.; Hak, S. C.; Zimmer, J. P.; Tanaka, E.; Frangioni, J. V.; Bawendi, M. Compact Cysteine-Coated CdSe(ZnCdS) Quantum Dots for in Vivo Applications. J. Am. Chem. Soc. 2007, 129, 14530-14531.

(26) Williams, Y.; Sukhanova, A.; Nowostawska, M.; Davies, A. M.; Mitchell, S.; Oleinikov, V.; Gun'ko, Y.; Nabiev, I.; Kelleher, D.; Volkov, Y. Probing Cell-Type-Specific Intracellular Nanoscale Barriers Using Size-Tuned Quantum Dots. Small 2009, 5, 2581-2588.

(27) Tamang, S.; Beaune, G.; Texier, I.; Reiss, P. Aqueous Phase Transfer of InP/ZnS Nanocrystals Conserving Fluorescence and High Colloidal Stability. ACS Nano 2011, 5, 93929402. 
(28) Mattoussi, H.; Mauro, J. M.; Goldman, E. R.; Anderson, G. P.; Sundar, V. C.; Mikulec, F. V; Bawendi, M. G. Self-Assembly of CdSe-ZnS Quantum Dot Bioconjugates Using an Engineered Recombinant Protein. J. Am. Chem. Soc. 2000, 122, 12142-12150.

(29) Uyeda, H. T.; Medintz, I. L.; Jaiswal, J. K.; Simon, S. M.; Mattoussi, H. Synthesis of Compact Multidentate Ligands to Prepare Stable Hydrophilic Quantum Dot Fluorophores. J. Am. Chem. Soc. 2005, 127, 3870-3878.

(30) Sukhanova, A.; Even-Desrumeaux, K.; Chames, P.; Baty, D.; Artemyev, M.; Oleinikov, V.; Nabiev, I. Engineering of Ultra-Small Diagnostic Nanoprobes through Oriented Conjugation of Single-Domain Antibodies and Quantum Dots. Protoc. Exch. 2012, DOI: $10.1038 /$ protex.2012.042.

(31) Sukhanova, A.; Even-Desrumeaux, K.; Kisserli, A.; Tabary, T.; Reveil, B.; Millot, J.-M.; Chames, P.; Baty, D.; Artemyev, M.; Oleinikov, V.; et al. Oriented Conjugates of SingleDomain Antibodies and Quantum Dots: Toward a New Generation of Ultrasmall Diagnostic Nanoprobes. Nanomedicine Nanotechnology, Biol. Med. 2012, 8, 516-525.

(32) Sayevich, V.; Cai, B.; Benad, A.; Haubold, D.; Sonntag, L.; Gaponik, N.; Lesnyak, V.; Eychmüller, A. 3D Assembly of All-Inorganic Colloidal Nanocrystals into Gels and Aerogels. Angew. Chemie Int. Ed. 2016, 55, 6334-6338.

(33) Page, R. C.; Espinobarro-Velazquez, D.; Leontiadou, M. A.; Smith, C.; Lewis, E. A.; Haigh, S. J.; Li, C.; Radtke, H.; Pengpad, A.; Bondino, F.; et al. Near-Unity Quantum Yields from Chloride Treated CdTe Colloidal Quantum Dots. Small 2015, 11, 1548-1554.

(34) Chen, O.; Zhao, J.; Chauhan, V. P.; Cui, J.; Wong, C.; Harris, D. K.; Wei, H.; Han, H.-S.; Fukumura, D.; Jain, R. K.; et al. Compact High-Quality CdSe-CdS Core-Shell Nanocrystals with Narrow Emission Linewidths and Suppressed Blinking. Nat. Mater. 2013, 12, 445-451. 
(35) Boldt, K.; Kirkwood, N.; Beane, G. A.; Mulvaney, P. Synthesis of Highly Luminescent and Photo-Stable, Graded Shell $\mathrm{CdSe} / \mathrm{Cd}_{\mathrm{x}} \mathrm{Zn}_{1-\mathrm{x}} \mathrm{S}$ Nanoparticles by In Situ Alloying. Chem. Mater. 2013, 25, 4731-4738.

(36) Pu, C.; Qin, H.; Gao, Y.; Zhou, J.; Wang, P.; Peng, X. Synthetic Control of Exciton Behavior in Colloidal Quantum Dots. J. Am. Chem. Soc. 2017, 139, 3302-3311. 